\title{
Modified blanket cooling manifold system for ITER
}

\author{
A.Furmanek*(1), P. Lorenzetto(2), C. Damiani(2) \\ 1. Max-Planck Institut für Plasmaphysik, Boltzmannstr. 2, 85748 Garching, Germany \\ 2. Fusion for Energy, C/ Josep Pla, 2. Torres Diagonal Litoral B3, 08019 Barcelona, Spain
}

\begin{abstract}
$\underline{\text { Abstract }}$
The modular ITER shielding blanket is the innermost part of the reactor directly exposed to the plasma. Its high thermal and nuclear loaded blanket modules (BMs) are water-cooled components. The cooling water is supplied to the BMs by a set of stiff inlet and outlet manifolds mechanically attached to the inner wall of vacuum vessel (VV). The ITER reference design of the blanket cooling manifold (BCM) presents some concerns namely: huge reaction forces at the end supports on the $\mathrm{VV}$, high operating stresses at certain locations on the manifolds the difficulty of localizing possible water leakages and the inability to repair the components remotely in a case of manifold damage.
\end{abstract}

To overcome these problems, a proposal for a new manifold concept has been developed within the European Fusion Development Agreement - Central Support Unit in Garching in the frame of the ITER design review.

The original stiff welded structure has been replaced by a bundle of elastic single seamless pipes supplying the cooling water to each module.

Based on FEM and CFD calculations the shape and diameter for the pipes were chosen to cope with thermal differential expansion as well as with electro-magnetic (EM) loads and pressure drop requirements. An attachment system was developed to enable precise positioning and fixing of the manifold segments onto the inner wall of the VV. Additionally a remote handling $(\mathrm{RH})$ procedure for the removal and installation sequences was created for the case of manifold damage during operation. This procedure is based on the currently planned RH equipment such as the maintenance cask and the in-vessel transporter (IVT).

Keywords: ITER; Blanket manifold; Attachment system; Remote handling

\section{Introduction}

The ITER reference BCM system [1,2] is a set of welded steel profiles forming multiple poloidal channels and toroidal segments attached mechanically onto the inner wall of $\mathrm{VV}$. The entire manifold system consists of $20^{\circ}$ toroidal sectors located around the plasma chamber. In each sector there is an inlet and outlet manifold in $10^{\circ}$ spacing with two main parts - the inboard and outboard one. Due to the $20^{\circ}$ toroidal segmentation of the inboard blanket there are recesses in the back side of these blanket modules for the inboard manifolds. The outboard manifolds are routed between the $10^{\circ}$ poloidal rows of blanket modules but the triangular voids there are to narrow and additional cut-outs in the modules are necessary.

Each single poloidal channel in the manifold is connected on its upper end with a separate feeder pipe. All the pipes are routed out from the VV through the upper port (UP) and channeled on its end upwards from the port duct through special twin 
chimneys. The manifold channels supply the modules with cooling water through elastic $S$ shaped branch pipes routed in corresponding recesses in the back part of the BMs. Every manifold channel feed a group of 2-4 modules.

To allow the replacement of modules there are toroidal voids in the blanket located in the vicinity of the equatorial and upper ports. The manifold toroidal segments - so called filler shields (FSs) - fill this space between the blanket modules.

Due to the inconsistency between the regular $10^{\circ}$ outboard blanket segmentation and the size of the equatorial port openings, it is necessary to have at these openings a joggle in the outboard manifolds and the FSs are making this offset possible. Fig. 1 shows the $20^{\circ}$ standard manifold sector.

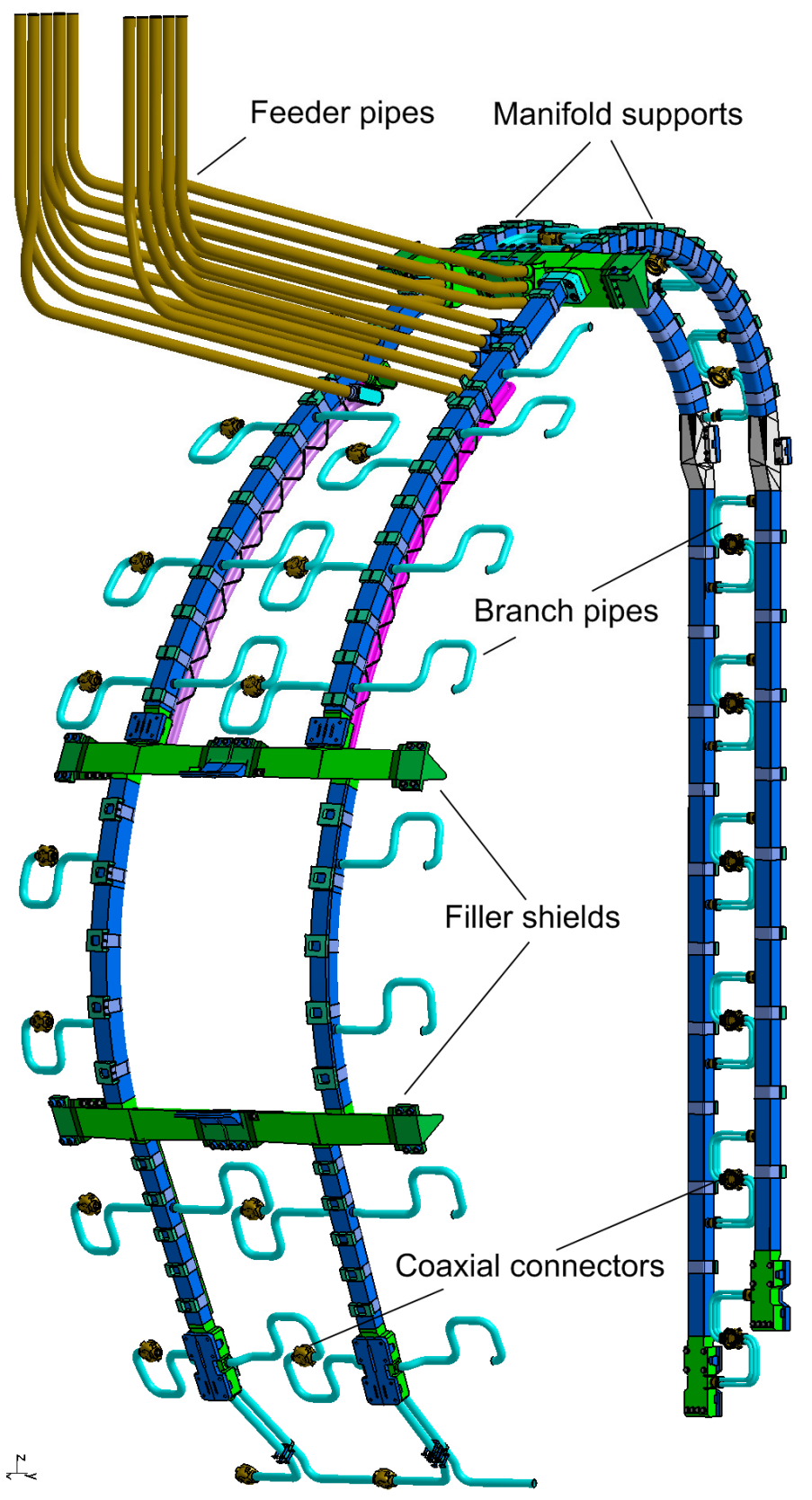

Fig.1 Reference design of a $20^{\circ}$ blanket cooling manifold sector 
The poloidal parts of the manifolds are stiff fastened at the base and the endings of the FSs are elastically anchored onto the VV.

Due to the temperature differences between the manifold and the VV there are axial forces of 90 tons in each of the 9 outlet manifold profiles and corresponding reactions on the VV. The stresses in the manifold caused by axial forces are on an acceptable level; however, as indicated in [3], the non-axial force transition in the joggles creates higher stresses in the connection with the FSs. The stresses are particularly concerning in their end anchors and could cause fatigue failure, in spite of using materials stronger than the ITER standard grade 316 SS for each of the 108 elastic FS endings. The manifold curvature induces forces normal to the VV wall, thus sliding supports in between are necessary. In the top region, despite installing them every $25 \mathrm{~cm}$, there are reaction forces up to 25 tons per support. This can change dramatically if sliding in the supports does not occur. In-plane forces up to 33 tons may result from unwanted friction.

Due to the large cross section of the massive welded manifold structure there are large EM radial forces (perpendicular to the manifold axis) in the range of $12 \mathrm{ton} / \mathrm{m}$ during certain events. Thus, manifold supports are necessary every $33 \mathrm{~cm}$ for the inboard and outboard region.

In the case of a leak during plasma operation it would be problematic to localize a leak in the cooling system due to the restricted access for leak testing and because one single manifold channel is connected with a group of 2-4 BMs. In addition in such a parallel cooling circuit it is also challenging to control the required cooling water mass flow rate in each separate module supplied from the one single inlet manifold channel.

There is an additional issue that the highest loaded filler shields are subject to extreme neutron irradiation and corresponding material damage, thus it will be difficult or impossible to repair them in situ after a certain period of operation. Also it will be impossible to access some welds particularly on the back site of the manifold for repair with RH tools. After a detailed analysis it was found [4], that the reference design of ITER cooling manifold can not be maintained by RH methods.

Considering all of the concerns above an ITER Issue Card was launched [5] with some proposals for optimization or redesign of the ITER BCM system.

\section{Modified manifold concept}

In the frame of the ITER Design Review the development work has been undertaken in Europe with the objective of demonstrating viable solutions and decreasing the fabrication and maintenance costs for the BCM system. The work conducted aimed at developing a design that eliminates the disadvantages of the reference BCM system and at the same time has no or minimal impact on the other components of the reactor. To replace the stiff welded structure a bundle of separate seamless pipes located parallel in the existing recesses of the BMs and fastened mechanically onto the inner wall of $\mathrm{VV}$ using a number of sliding supports has been proposed. Every pipe is designed to feed one single $\mathrm{BM}$ and is routed all the way from the chimney bulkhead to the coaxial connector (CC) on the module.

Two bundles of 24 pipes with two branches, with 9 and 15 pipes feeding the inboard and outboard modules, respectively, form each of the single $20^{\circ}$ standard sectors of the modified BCM system. One of the bundles acts as the inlet and the second one as the outlet part of the manifold sector. To allow unrestricted thermal differential expansion 
between the manifold pipes and the VV the end regions of the pipes are bent. In addition the routing of the outboard manifold pipes from the VV into the UP duct includes an additional bend which allows simplified bending in the module region. The design of the modified BCM is shown in the Fig. 2. For the installation and maintenance reasons each pipe consists of two parts joined smoothly together using an orbital weld in the vicinity of the UP opening. The routing of the pipes in this region ensures an access for the RH welding and cutting tools.

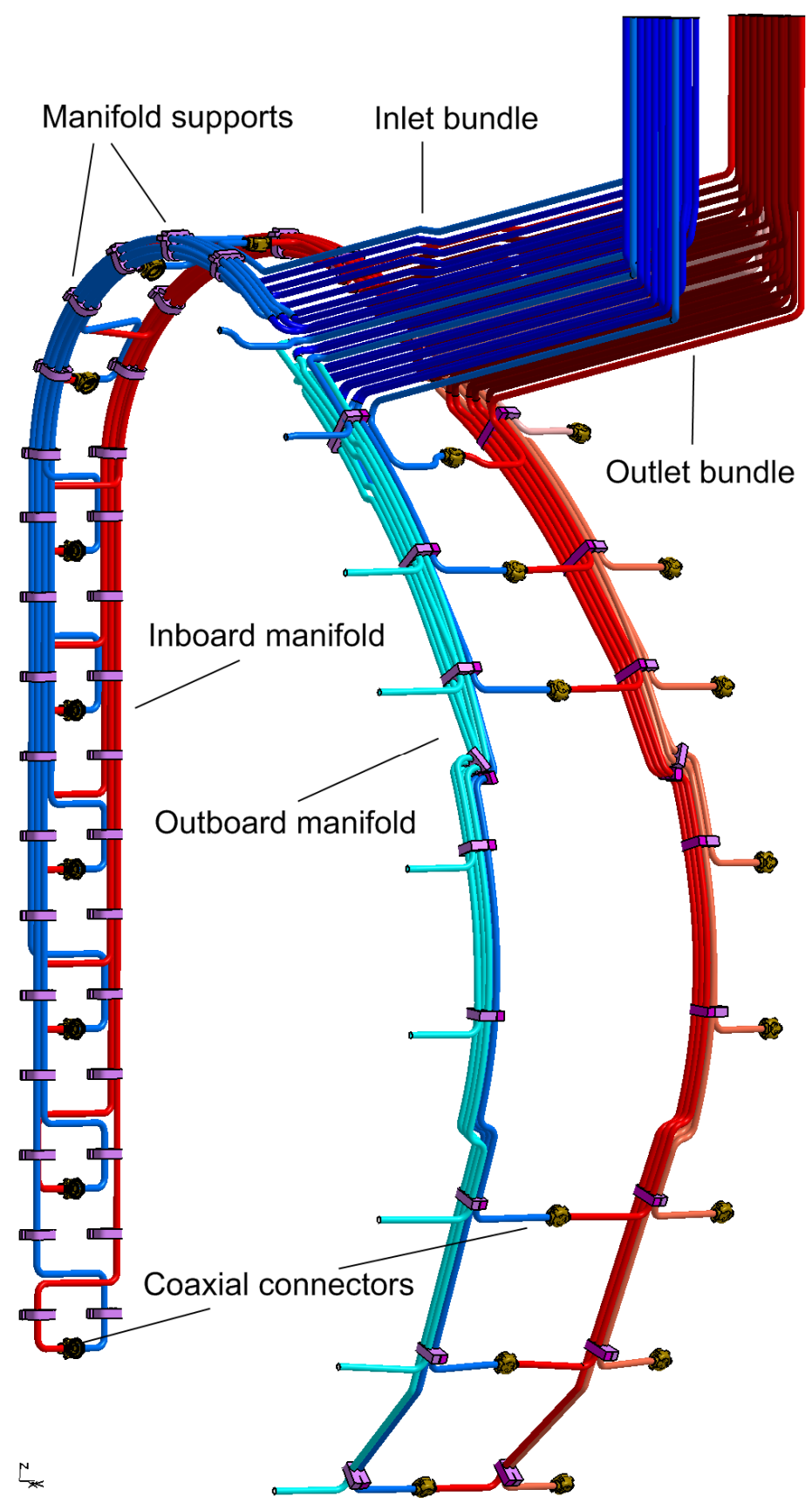

Fig. 2 Modified design of the blanket cooling manifold 
One of the issues during the development process of the modified BCM was finding a good balance between the mechanical (e.g. stiffness) and hydraulic (e.g. pressure drop) properties of the manifold components. For this reason, some iterations of the EM loads assessment [6] the FEM computations [7] and the hydraulic analysis have been performed, to find the proper cross section for the manifold pipes and the right spacing and size for the manifold sliding supports.

For the EM loads analysis the worst case scenarios for the off-normal events were considered as follow: plasma thermal quench during a type II fast downward vertical displacement event (VDE), current quench during centered disruption of type II and toroidal field coil fast discharge with the time constant of $10 \mathrm{~s}$.

For the EM calculations a $10^{\circ}$ model of the ITER toroidal sector with the shielding blanket modules and modified manifold was created. In the last iteration the results obtained for the 44,5 x 38,7 mm dia. ISO standard pipes show, that the highest EM loads in the range of $11 \mathrm{kN} / \mathrm{m}$ occur during the thermal quench and act on the inboard pipes supplying the blanket modules in the equatorial region.

For the FEM calculations the following mechanical, thermal and EM loads parameters acting on the longest inboard and outboard manifold pipes have been considered:

- sliding support every $60 \mathrm{~cm}$,

- $1 \mathrm{~mm}$ displacement in all directions applied at the bottom end of pipe (inaccurate assembling)

- 4,4 MPa pressure acting on the internal surface of the pipe,

- $50^{\circ} \mathrm{C}$ temperature difference between the pipe and the support,

- $\quad 11 \mathrm{kN} / \mathrm{m}$ for the inboard and $5 \mathrm{kN} / \mathrm{m}$ for the outboard pipe (evenly distributed EM load for conservative calculation).

In the results presented in Table 1 the maximum peak stresses are below the membrane stress limit Sm for the ITER standard grade 316 SS and the analyzed structure can be considered safe with margin.

\begin{tabular}{|c|c|c|c|c|c|c|c|c|c|c|}
\hline \multirow{3}{*}{$\begin{array}{l}\begin{array}{l}\text { Support } \\
\text { spacing }\end{array} \\
60 \mathrm{~cm}\end{array}$} & \multirow{3}{*}{$\frac{\mathscr{n}}{\stackrel{\Xi}{\Xi}}$} & \multicolumn{9}{|c|}{ LOAD CASE } \\
\hline & & \multicolumn{4}{|c|}{$\begin{array}{c}\text { Inaccurate assembling } \\
(\mathbf{m m})\end{array}$} & \multirow{2}{*}{$\begin{array}{c}\mathbf{P R} \\
\begin{array}{c}\mathrm{p}= \\
4.4 \mathrm{MPa}\end{array}\end{array}$} & \multirow{2}{*}{$\begin{array}{c}\mathbf{T H} \\
\Delta \mathrm{T}= \\
50^{\circ} \mathrm{C}\end{array}$} & \multirow{2}{*}{$\begin{array}{c}\mathbf{E M} \\
\mathrm{q}= \\
11 \mathrm{kN} / \mathrm{m}\end{array}$} & \multirow{2}{*}{$\begin{array}{c}\mathbf{E M} \\
\underset{\mathrm{q}=}{5 \mathrm{kN} / \mathrm{m}}\end{array}$} & \multirow{2}{*}{$\begin{array}{c}\mathbf{A L L} \\
\mathrm{p}, \Delta \mathrm{T}, \mathrm{q}, \\
{[1,1,1]}\end{array}$} \\
\hline & & $1,0,0$ & $0,1,0$ & $0,0,1$ & $1,1,1$ & & & & & \\
\hline \multirow{2}{*}{$\begin{array}{l}\text { Inboard } \\
\text { pipe }\end{array}$} & $\begin{array}{l}\max \\
\mathrm{u}_{\text {sum }} \\
(\mathrm{mm})\end{array}$ & 1.03 & 1.1 & 1.00 & 1.8 & 0.60 & 10.1 & 1.4 & - & 10.9 \\
\hline & $\begin{array}{l}\max \\
\sigma_{\text {eqv }} \\
(\mathrm{MPa})\end{array}$ & 33.6 & 21.2 & 6.1 & 44.7 & 45.0 & 28.6 & 73.0 & - & 89.8 \\
\hline \multirow{2}{*}{$\begin{array}{l}\text { Out- } \\
\text { board } \\
\text { pipe }\end{array}$} & $\begin{array}{l}\max \\
u_{\text {sum }} \\
(\mathrm{mm})\end{array}$ & 1.0 & 1.0 & 1.0 & 1.7 & 0.43 & 7.2 & - & 0.8 & 6.8 \\
\hline & $\begin{array}{l}\max \\
\sigma_{\text {eqv }} \\
(\mathrm{MPa})\end{array}$ & 14.6 & 19.4 & 9.4 & 37.8 & 38.7 & 35.0 & - & 83.9 & 136.3 \\
\hline
\end{tabular}

Table 1. FEM calculation results for the longest inboard and outboard pipes 
The main hydraulic requirement for the in-vessel cooling circuit is to keep the pressure drop low. For this reason the hydraulic analysis of 3 pipes with the highest pressure drop from the inlet part of manifold has been performed. The longest inboard and outboard pipe feeding the modules No.1 and No.18 respectively and the pipe with the highest mass flow rate of cooling water which feeds the outboard module No.15 have been analyzed. In the last iteration an inner diameter of $38,7 \mathrm{~mm}$ was considered. The calculation results are shown in Tab. 2.

\begin{tabular}{|c|c|c|c|}
\hline $\begin{array}{c}\text { Pipe for } \\
\text { module No. }\end{array}$ & $\begin{array}{c}\text { Mass flow rate } \\
{[\mathrm{kg} / \mathrm{s}]}\end{array}$ & $\begin{array}{c}\text { Average velocity } \\
{[\mathrm{m} / \mathrm{s}]}\end{array}$ & $\begin{array}{c}\text { Pressure drop } \\
{[\mathrm{MPa}]}\end{array}$ \\
\hline 1 & 6,365 & 5,64 & 0,078 \\
\hline 15 & 8,86 & 7,85 & 0,1122 \\
\hline 18 & 7,39 & 6,55 & 0,0944 \\
\hline
\end{tabular}

Table 2. Hydraulic analysis parameter and results

The computed values of cooling water velocity and pressure drop in the worst case pipes are similar to the average numbers agreed for the reference manifold design [2]. Due to the smooth curvatures of the streaming and the finally considered pipe inner diameter the pressure drop for both inlet and outlet branches is in the range of $20 \%$ of the pressure drop limit of $1 \mathrm{MPa}$ for the whole in-vessel circuit. Taking into account the suggested 0,5 MPa pressure drop in the cooling system of a BM, the proposed solution provides a substantial engineering margin.

In spite of keeping the joggles in the routing of the pipes the proposed BCM system eliminates the disadvantages of the reference solution and the only impacts of the modified design on the other components are smaller and less complicated recesses behind the BMs and reduced number and size of manifold supports on the VV.

\section{Manifold attachment system}

To ensure precise positioning of the modified BCM onto the inner VV wall a special attachment system with an installation and removal sequence has been developed. The primary objective for the attachment system was to ensure that the maximal positioning inaccuracy for the $\mathrm{CC}$ does not exceed $1 \mathrm{~mm}$ in all directions [2]. Due to the welding tolerance for $\mathrm{VV}$ of $\pm 10 \mathrm{~mm}$ the attachment points for all the In-Vessel components have to be customized on the base of a laser survey, where a final precision of $0,2 \mathrm{~mm}$ is achievable.

The proposed attachment system uses a number of supports along the pipe bundles for the manifold fastening onto the VV wall. The attachment sockets for the blanket electrical straps are used as the reference points for positioning of CCs during the attachment process. These components themselves have to be customized or corresponding attachment pads for them have to be fastened (screwed or welded) onto the VV wall. Fig. 3 shows the design of a single manifold support fastened to the attachment pad on the VV wall. 


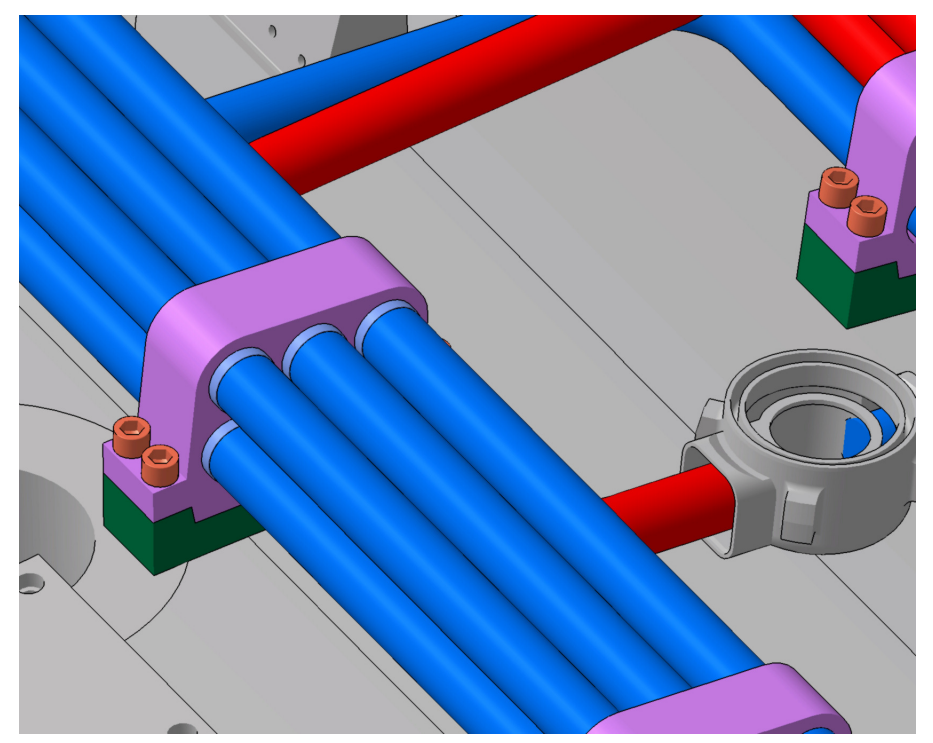

Fig. 3 Manifold sliding support

For the precise positioning of the $\mathrm{CC}$ a special $\mathrm{CC}$ holder has been proposed (refer to Fig. 4) with attachment interfaces to the neighboring sockets for the electrical straps.

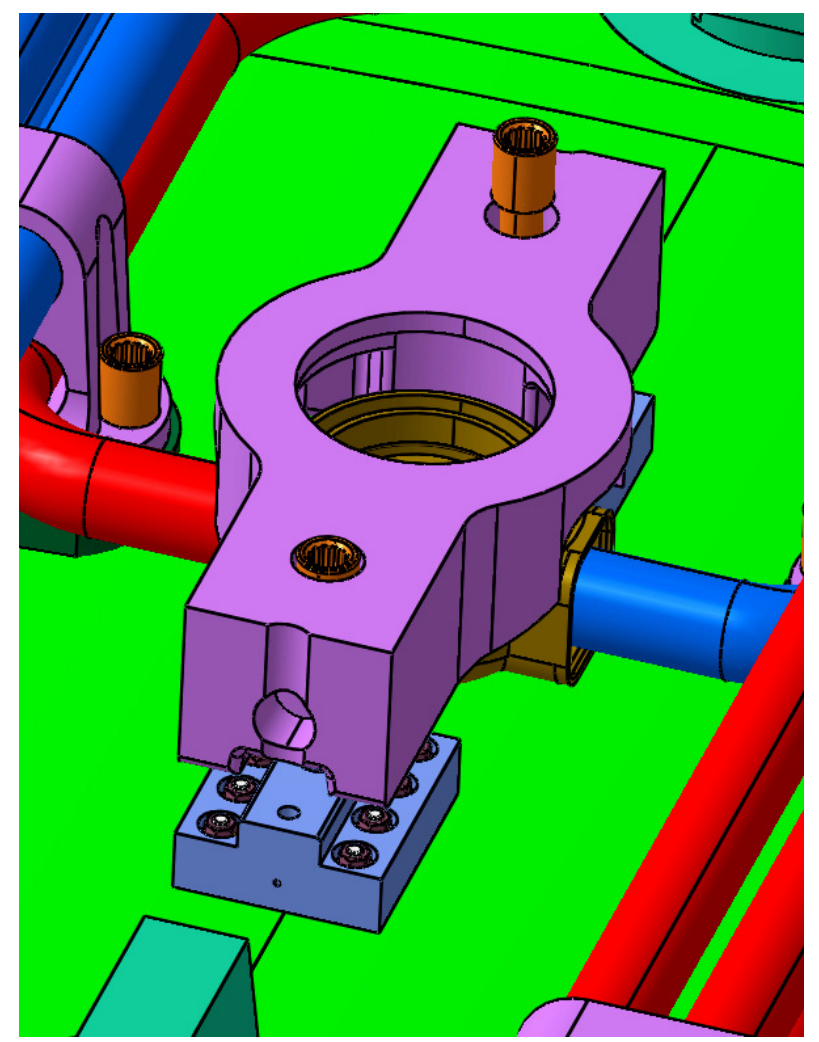

Fig. 4 Coaxial connector holder

In addition, carrying frames have been designed to allow the attachment of the inboard and outboard manifold assemblies during installation. The carrying frames have identical RH gripping interface (size and position) to the BMs and so they can be used for the installation and maintenance of damaged manifolds using IVT (developed for the RH of the BMs). Fig. 5 illustrates such frame with the inboard manifold sector. 


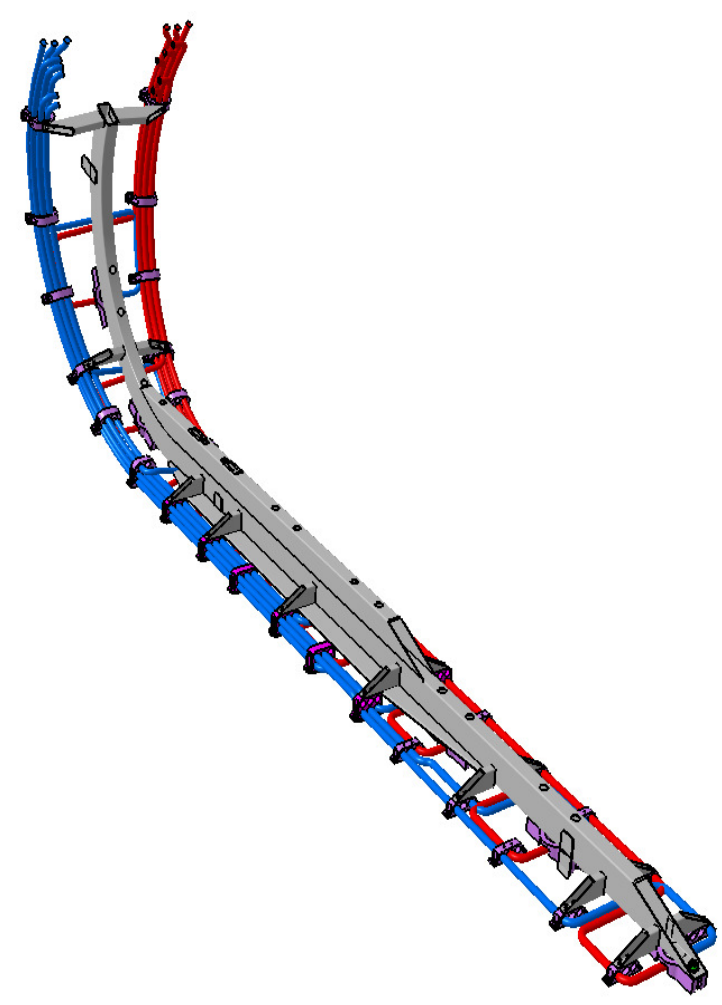

Fig. 5 Carrying Frame with the inboard manifold assembly

The installation sequence using the carrying frame is as follow:

- the manifold sector with CCs and supports is mounted in the workshop on the carrying frame using bolts and CC holders,

- this assembly is positioned correctly on the VV using guiding sheets on the frame that slide on the electrical strap sockets (ESS),

- the CC holders are bolted to the ESS bringing the CCs into the correct position,

- the two parts of manifold pipes (VV and UP parts) are joined using an orbital welder,

- the manifold supports are bolted to the attachment pads on the VV,

- the carrying frame is unscrewed from the CC holders and removed from the VV,

- the CC holders are unbolted from the ESS and retracted from the VV.

The described above attachment system and installation method ensure that the positioning of the CCs is accomplished with a precision not exceeding the required 1 $\mathrm{mm}$ tolerance in all directions.

\section{Manifold remote handling}

The ITER BCM is a RH Class 3 component, which means that it is not expected to be replaced remotely however it should be possible.

The recommendations for the development of a modified manifold from the RH study [4] are as follow:

- the manifold should withstand the worst foreseeable loads to exclude the need for its full replacement,

- its design should utilize circular pipes with reduced number of fully accessible welds for the RH tools.

The presented above design of the modified BCM fulfills all the recommendations and in addition it is designed to facilitate RH replacement. The preliminary study shows that 
the RH replacement for the full length manifold sectors would require a maneuverability of the currently developed RH equipment (the in-vessel transporter, IVT) that is probably not achievable. Therefore, the manifold can be designed in two poloidal segments which will allow simpler trajectories for the IVT manipulator.
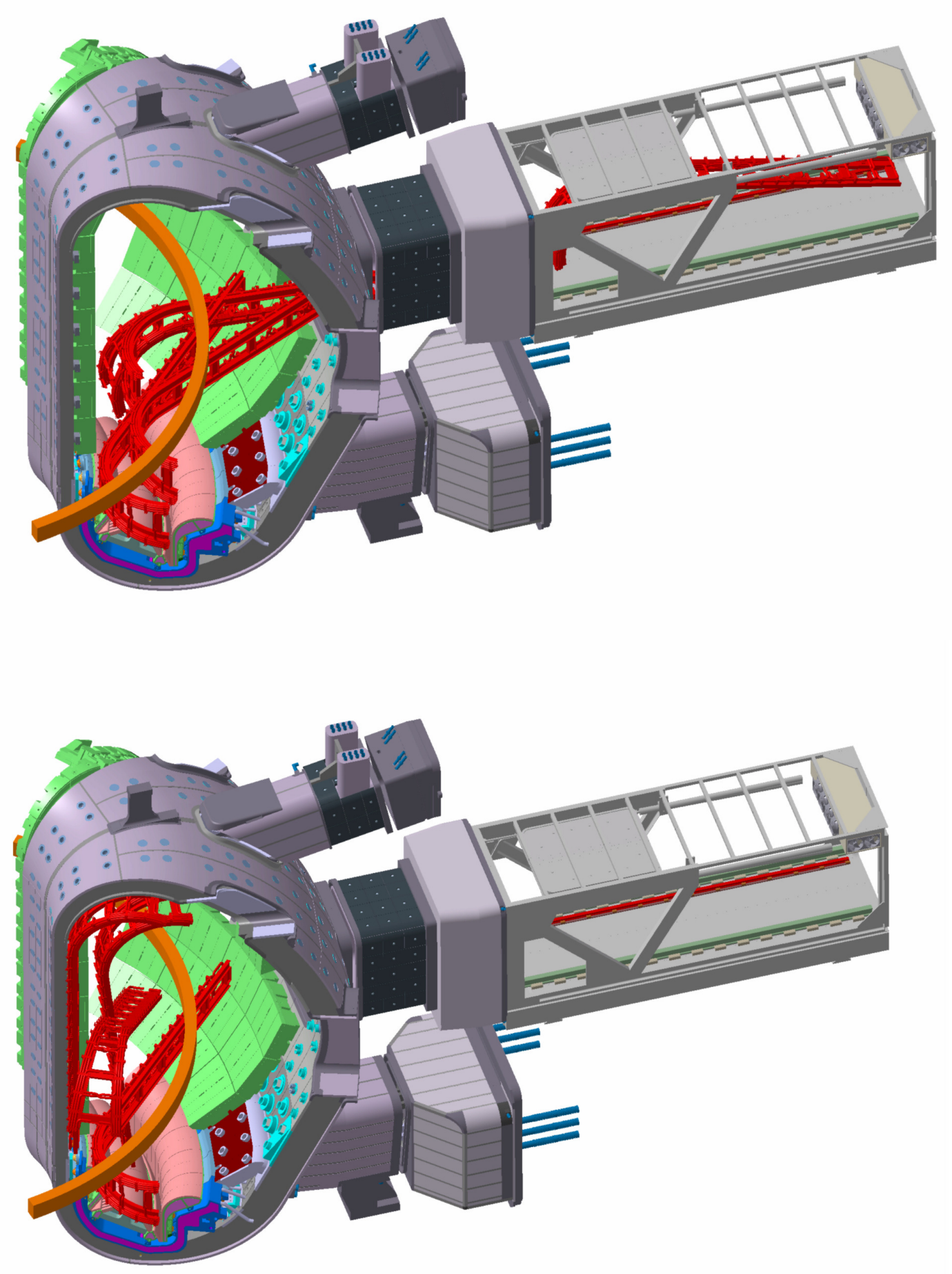

Fig. 6 RH study of the inboard manifold sector in the VV and transfer cask 


\section{Conclusions}

The modified design of the BCM developed in Europe utilizes separate elastic seamless pipes to supply of BMs with cooling water. The pipes form bundles located in existing recesses behind the BMs and are mechanically attached to the inner wall of the VV using sliding supports. The size and geometry of the pipes was determined to fulfill the hydraulic requirements and to cope with the worst load cases. The proposed attachment system with suitable equipment ensures precise positioning and proper fastening of the manifold onto the VV wall. In the very unlikely case of the manifold leakage the currently planned RH equipment can be used for the replacement of the damaged BCM sectors. Utilizing of standard seamless pipes contributes to keeping the fabrication costs down.

\section{Acknowledgments}

This work, supported by the European Communities, was carried out within the framework of the European Fusion Development Agreement. The views and opinions expressed herein do not necessarily reflect those of the European Commission.

\section{References}

1. F. Elio, K. Ioki, M. Yamada, H. Yoshimura, Design of blanket cooling manifold system for ITER-FEAT; Fus. Eng. Des. 58-59; November 2001, p. 579-583.

2. DDD, Chapter 16, Blanket, ITER_D_22F3M6_2_0, August 2004.

3. A. D. McCallum, Analysis of revised outboard manifold design, September 2003, EFDA Contract No.: FU05-CT2002-00133.

4. A. Tessini, Assessment of the Repair \& Replacement Ability of the ITER Blanket Water Cooling Manifold, September 2007 ITER D 28MXTK v1.2.

5. L. Jones, ITER Issue Card WBS-15, Rigid Blanket Manifolds - Optimization or ReDesign to Flexible System

6. M. Roccella, A. Marin, F. Lucca, G, Ramogida, Preliminary Electro-Magnetic Load Analysis for the Blanket Manifold Pipe Concept for ITER, Frascati, December 2007. 7. G. Krzesinski, Preliminary mechanical analysis of a blanket manifold concept for ITER, Warsaw, March 2008 